\title{
Uma Abordagem V2X para Disseminação de Dados em Redes Veiculares
}

\author{
Luiz Henrique C. Silva ${ }^{1}$, Raquel A. F. Mini ${ }^{1}$, Felipe D. Cunha ${ }^{1}$ \\ ${ }^{1}$ Departamento de Ciência da Computação \\ Pontifícia Universidade Católica de Minas Gerais (PUC-Minas) \\ lhcsilva@sga.pucminas.br, \{raquelmini,felipe\} @pucminas.br
}

\begin{abstract}
Vehicular ad-hoc networks are a promising type of networks that allow communication between vehicles with the aim to promote safe and efficient traffic. During the trips, vehicles can communicate with each other and with other networks by interacting with them and with the cell phone network. The literature does not present many solutions that involve this existing infrastructure in the cities, and in order to disseminate data to a group of vehicles, it is interesting to know better the global behavior of the scenario. So, in this work, we proposed a protocol to disseminate data that select the vehicle to relay the information according to its position in relation to the destination of the message. Simulation results evaluated in scenarios of various densities have shown that it is possible to reduce the number of messages transmitted with a good delivery ratio.
\end{abstract}

Resumo. Redes Veiculares são um tipo promissor de rede ad-hoc que permite a comunicação entre veículos, com o objetivo de promover um tráfego seguro e mais eficiente. Durante suas viagens, os veículos podem se comunicar uns com os outros e com outras redes, por meio das interações entre eles e a rede celular. A literatura não apresenta muitas soluções que envolvam esta infraestrutura existente nas cidades, e para efetuar a disseminação de dados para um grupo de veículos é interessante conhecer melhor o comportamento global do cenário. Assim, neste trabalho é proposto um protocolo para disseminar os dados, que seleciona veículos para retransmitir a informação de acordo com suas posições em relação ao destino da mensagem. Resultados de simulação avaliados em cenários de diferentes densidades mostraram que é possivel reduzir o número de mensagens transmitidas mantendo uma boa taxa de entrega.

\section{Introdução}

A crescente demanda por comunicação no século XXI tem impulsionado os estudos de redes sem fio para aplicações que melhoram a qualidade de vida. Na última década, a comunicação em redes veiculares tem despertado interesse da academia, indústria e órgãos governamentais [Peng et al. 2018]. Este interesse ocorre devido ao grande potencial que as redes veiculares têm de aumentar a segurança e desempenho no trânsito. Inseridas no campo das redes móveis, as redes veiculares são formadas por veículos equipados com dispositivos de comunicação sem fio, processamento e sensoriamento.

Embora as redes veiculares apresentem novas oportunidades para o provimento de serviços inovadores, também apresentam novos desafios. A alta mobilidade dos nós 
em redes veiculares caracteriza a dinamicidade da topologia dessas redes, trazendo consigo a necessidade do fornecimento de informação em tempo real para os envolvidos na comunicação. Duas áreas de aplicações que se destacam no contexto das redes veiculares são as de segurança no trânsito e de melhoria no desempenho do trânsito.

Um exemplo de aplicação para segurança no trânsito por meio das redes veiculares é a notificação de eventos de segurança. Estes eventos podem ser naturais (oriundos de mudanças climáticas em virtude das estações do ano e das características do ambiente) ou ainda não-naturais (provenientes, por exemplo, da negligência dos condutores de veículos). Aplicações de notificação como esta exigem da rede a atividade de disseminação de dados que consiste no envio da mensagem por um veículo origem e a entrega desta mensagem a todos os veículos na região de interesse (Region of Interest - RoI).

A atividade de disseminação de dados também pode ser utilizada para o aumento do desempenho no trânsito. Uma das principais causas dos congestionamentos é a restrição espacial das vias, assim sendo, uma boa gestão do recurso espaço pode ser feita por meio da comunicação entre os veículos informando os condutores para impedir que situações mais críticas como acidentes ocorram ou, se ocorrerem, pode reduzir os prejuízos para o desempenho do trânsito. Neste ponto, a disseminação de dados tem um papel fundamental, o de levar a informação ao maior número de condutores no menor espaço de tempo, permitindo que os condutores tomem decisões inteligentes evitando acidentes e congestionamentos. Independente da aplicação, a atividade de disseminação de dados é fortemente influenciada pela densidade de veículos no cenário, e dois cenários opostos são bastante comuns nas grandes cidades.

Num primeiro cenário, de baixas densidades de veículos, os nós apresentam-se provavelmente mais distantes e, além de se encontrarem menos, o que proporciona um número menor de conexões, têm liberdade para alcançarem velocidades mais altas, provocando conexões por tempos mais curtos que a média (desconexões mais constantes). A ausência de conexões ou ainda as conexões por curtos espaços de tempo são características que identificam o problema de partições na rede.

Num segundo cenário, de altas densidades de veículos, os nós aglomeram-se mais facilmente, além disso, devido às restrições espaciais, movem-se mais lentamente, permitindo conexões mais longas entre si, apesar de naturalmente curtas. Este cenário é facilmente percebido em horários de pico, durante o início da manhã e o fim da tarde de dias úteis, caracterizado pelos constantes congestionamentos.

Este cenário de altas densidades apresenta ainda complicações relacionadas aos recursos de rede. Devido à comunicação em redes veiculares ser via broadcast, o número de transmissões feitas numa disseminação de dados pode aumentar conforme a densidade de veículos. Por exemplo, a decisão sobre retransmitir deve filtrar bem os veículos responsáveis pela tarefa para impedir o crescimento do número de mensagens duplicadas, reduzindo o consumo do recurso de rede e impedindo que ocorram colisões e perdas de dados. Este problema é conhecido na literatura como broadcast storm.

Nestes dois cenários comuns encontrados nas grandes cidades, as redes veiculares tem um papel muito promissor na melhoria da segurança no trânsito. O trabalho de [Toor et al. 2008] mostrou que aplicações de segurança em redes veiculares podem reduzir acidentes em cerca de $60 \%$ se capazes de informar os condutores acerca de eventos 
relacionados com cerca de 0,5 segundo de antecedência. Em 2013, a Organização Mundial de Saúde publicou um relatório [W.H.O. 2013] que apresenta o atual estado global da segurança no trânsito. De acordo com o relatório, cerca de 1.24 milhão de pessoas morrem em acidentes de trânsito todos os anos. Garantir a entrega de notificações de segurança em tempo hábil apresenta potencial para uma grande redução nestes números. A atividade que dá suporte a esta entrega é a disseminação de dados.

Muitos dos estudos em redes veiculares voltados para disseminação de mensagens de segurança têm concentrado esforços na comunicação V2V primariamente. Embora o cenário ideal, do ponto de vista financeiro, para implantação de redes veiculares não envolva aquisição de infraestrutura, a utilização da infraestrutura existente pode apresentar grandes avanços sem custo adicional, integrando redes celulares e de computadores convencionais, passos necessários para o futuro da Internet das Coisas.

Diante da necessidade de segurança no trânsito e das emergentes redes celulares de $5^{\text {a }}$ Geração, este trabalho propõe o TBD - Trajectory Based Dissemination, um novo protocolo de disseminação de dados em cenários de densidades variadas. O objetivo do TBD é utilizar a rede celular como auxílio para o conhecimento da rede, permitindo que se defina uma trajetória para guiar a disseminação da mensagem da origem até a região de interesse. Através de resultados de simulação em diferentes configurações de densidades foi possível verificar uma redução no número de mensagens transmitidas, mantendo uma boa taxa de entrega.

O restante deste artigo está organizado da seguinte forma: a Seção 2 apresenta trabalhos que tratam os problemas da disseminação de dados encontrados na literatura. A solução proposta neste trabalho é detalhada na Seção 3 . E na Seção 4 é descrito o processo de avaliação de desempenho, bem como a discussão dos resultados. Por fim, a Seção 5 apresenta as conclusões deste estudo e dá direções para trabalhos futuros.

\section{Trabalhos Relacionados}

A forma mais simples conhecida para disseminação de dados em redes veiculares é através do algoritmo de flooding, como mostrado por [Tseng et al. 2002]. Neste algoritmo, uma fonte $S$ inicia a transmissão de uma mensagem $m$ a todos os veículos no seu raio de alcance. Cada veículo alcançado por $S$ efetua a retransmissão de $m$ também a todos que estão em seu raio de alcance. Estas retransmissões ocorrem sucessivamente até que a rede seja inundada com os pacotes. Apesar de simples, essa abordagem é genérica, desconsidera o tipo de mensagem e sofre com o problema de broadcast storm.

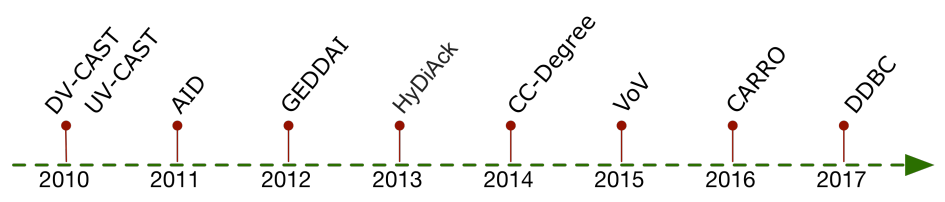

Figura 1. Protocolos de disseminação de dados com propósito semelhante apresentado nos últimos anos.

Outra solução proposta para VANETs é o protocolo AID (Adaptive approach for Information Dissemination) [Bakhouya et al. 2011]. Este é um protocolo descentralizado e adaptativo para a disseminação da informação. Nele, a decisão do veículo de encaminhar 
ou não o dado é feita baseada em probabilidade. Esta probabilidade é configurada baseada no número de vezes que o veículo recebe a mesma mensagem em um dado período de tempo. Desta forma, em cenários com alta densidade de tráfego, muitos veículos podem decidir a não enviar a informação baseado no fato de outros já terem a enviado. Entretanto, esta não é uma solução que lida com o problema das conexões intermitentes na rede.

Em [Akabane et al. 2016], os autores propuseram um protocolo que atua tanto em cenários urbanos como em rodovias. Além disso, o protocolo proposto CARRO (ContextAware Routing pROtocol) lida com os dois problemas clássicos de disseminação de dados, broadcast storm e partições na rede. A forma como abordaram a disseminação de dados envolve um mecanismo de difusão de beacons. Beacons são pacotes de dados de tamanho reduzidos que armazenam informações de contexto e são transmitidos a cada intervalo de tempo definido. As informações de contexto podem ser, por exemplo, a localização estimada por meio de sistemas de posicionamento global (Global Positioning System GPS), número de vizinhos, velocidade do veículo, etc. Estas informações permitem que os veículos façam estimativas acerca da topologia da vizinhança a um determinado número de saltos.

O processo de disseminação de dados ocorre a partir da observação da densidade de veículos no cenário, estimada por meio das informações contidas nos beacons. Em cenários densos de veículos o protocolo CARRO trata o problema de broadcast storm utilizando um mecanismo de supressão de broadcast chamado Zona de Preferência. Zonas de Preferência são setores na borda do raio de comunicação em que veículos localizados em seu interior são considerados melhores potenciais retransmissores de tal forma que bastando a retransmissão desses veículos a disseminação será eficiente. Por outro lado, em cenários pouco densos de veículos, o protocolo proposto pelos autores utiliza o mecanismo de armazena-e-encaminha para dar continuidade ao processo de disseminação.

Considerando as soluções que consideram ambos os cenários, o protocolo UVCAST [Viriyasitavat et al. 2010] é proposto considerando as diferentes condições de tráfego. Nele o veículo trabalha em dois estados: supressão de broadcast e armazenae-encaminha. Quando o veículo recebe uma mensagem de dados pela primeira vez, ele inicialmente verifica se é ou não um veículo de borda. Veículos de borda são aqueles que conectam dois ou mais componentes na rede. Assim, o UV-CAST assume que esses veículos têm grande probabilidade de encontrar novos veículos vizinhos. Neste caso, o veículo armazenará a mensagem e a encaminhará a cada novo encontro. Caso contrário, não sendo veículo de borda, ele executa os algoritmos de supressão de broadcast para encaminhamento da mensagem.

A Figura 1 apresenta alguns trabalhos desenvolvidos ao longo dos últimos anos acerca da disseminação de dados encontrados na literatura e detalhes acerca das propostas são resumidos na Tabela11. A grande maioria dos protocolos da literatura propõe soluções com a utilização da comunicação $\mathrm{V} 2 \mathrm{~V}$ apenas, poucos utilizando recursos infraestruturados para a comunicação. Além disso, ao efetuarem avaliações os protocolos consideram que todo o mapa é a região de interesse ou que o veículo origem da mensagem está no interior da região de interesse.

De forma diferente, neste trabalho a disseminação de dados é efetuada de forma a transmitir a mensagem até a região de interesse, e a partir daí disseminá-la para todos os 


\begin{tabular}{|c|c|c|c|c|c|c|}
\hline \multirow{2}{*}{ Protocolo } & \multirow{2}{*}{ Abordagem } & \multirow{2}{*}{ Beacons } & \multicolumn{2}{|c|}{ Problemas } & \multicolumn{2}{|c|}{ Cenário } \\
\hline & & & Broadcast storm & Partições na rede & Urbano & Rodovia \\
\hline DV-CAST & & & & & & \\
\hline [Tonguz et al. 2010] & estatística e contexto & 1-salto & $\checkmark$ & $\checkmark$ & - & $\checkmark$ \\
\hline UV-CAST & & & & & & \\
\hline Viriyasitavat et al. 2010] & estatística e contexto & 1-salto & $\checkmark$ & $\checkmark$ & $\checkmark$ & - \\
\hline AID & & & & & & \\
\hline Bakhouya et al. 2011 & estatística & - & $\checkmark$ & - & $\checkmark$ & - \\
\hline GEDDAI & & & & & & \\
\hline Villas et al. 2012 & geográfica & - & $\checkmark$ & - & $\checkmark$ & - \\
\hline HYDIACK & & & & & & \\
\hline Maia et al. 2013 & geográfica & 1 -salto & $\checkmark$ & $\checkmark$ & $\checkmark$ & - \\
\hline CC-DEGREE & & & & & & \\
\hline Cunha et al. 2014 & métricas sociais & 1-salto & $\checkmark$ & $\checkmark$ & $\checkmark$ & - \\
\hline VOV & & & & & & \\
\hline Maia et al. 2015 & geográfica & 1-salto & $\checkmark$ & $\checkmark$ & $\checkmark$ & - \\
\hline CARRO & & & & & & \\
\hline Akabane et al. 2016 & geográfica & 1 salto & $\checkmark$ & $\checkmark$ & $\checkmark$ & $\checkmark$ \\
\hline DDBC & & & & & & \\
\hline Costa et al. 2017 & grafos & 2-saltos & $\checkmark$ & - & $\checkmark$ & - \\
\hline
\end{tabular}

Tabela 1. Protocolos propostos na literatura nos últimos anos e suas características em termos abordagem, problemas tratados e cenários atendidos.

veículos no interior da região. Isso é feito a partir do conhecimento do comportamento global da cidade, obtido pela rede móvel.

\section{TBD - Trajectory Based Dissemination}

Nesta seção será descrito o protocolo TBD - Trajectory Based Dissemination, o qual considera a definição de uma trajetória para direcionar a disseminação de dados da origem até a região a ser alcançada. Para isso, o algoritmo tira proveito da comunicação com a rede celular para obter informações acerca da densidade da rede e assim, definir a trajetória da mensagem para a disseminação. Assim, vale ressaltar que:

(i) Os veículos estão equipados com dispositivo de comunicação sem fio com mesmo alcance de comunicação

(ii) Os veículos estão conectados à rede celular

(iii) Os veículos estão equipados com dispositivo GPS

(iv) A mensagem a ser transmitida leva a localização da região de interesse

(v) O veículo origem da mensagem está fora da região de interesse

Como requisito fundamental da disseminação, a mensagem deve alcançar os veículos no interior da região de interesse. Neste contexto, o TBD trabalha pra efetuar esta tarefa minimizando o consumo de recurso da rede. Assim, através de uma interação com a rede celular, o protocolo obtém informação da densidade de veículos por região, de forma a definir a trajetória que a mensagem irá percorrer até alcançar a região de destino. Esse conhecimento só é possível pois os veículos encaminham periodicamente sua localização para a rede celular, com o intuito de que se tenha ciência de todo o comportamento global dos veículos. Essa abordagem evita a troca constante de beacons entre os veículos, o que contribui para reduzir o consumo de recursos da rede. 
Como pode ser visto na Figura 2(a), a solução proposta divide a rede em células. Cada célula trata-se de um quadrado de lado equivalente ao tamanho do raio de comunicação. A partir desta divisão e dos envios de mensagens de localização de cada veículo, têm-se uma matriz de densidades. A periodicidade do envio destas mensagens pode ser definida de acordo com o tempo e também com a frequência de movimentação de cada veículo.

\begin{tabular}{|l|l|l|l|l|}
\hline 100 & 100 & 20 & 20 & 50 \\
\hline 50 & 20 & 100 & 20 & 20 \\
\hline 20 & 50 & 30 & 100 & 100 \\
\hline 20 & 30 & 50 & 20 & 30 \\
\hline 30 & 20 & 20 & 50 & 20 \\
\hline
\end{tabular}

(a) Matriz de densidades.

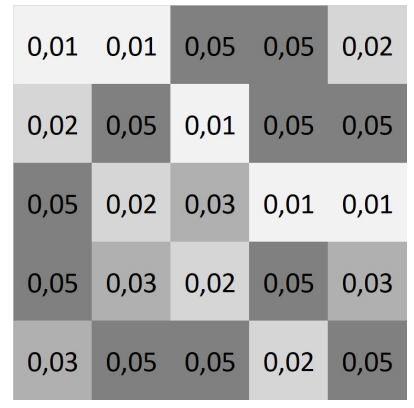

(b) Matriz de pesos.

Figura 2. (a) Exemplo de matriz de densidades, conforme submissões de veículos nas células. (b) Densidades convertidas em pesos, entrada para o algoritmo de Dijkstra.

Conhecendo a matriz de densidades, os veículos podem encontrar o caminho mais denso de veículos da origem até o destino da mensagem. Na Figura 2(b), pode-se verificar o valor das densidades invertidos, de forma que os mesmos possam ser usados como entrada para o cálculo do problema de encontrar o caminho mais curto entre dois pontos. Assim, densidades maiores serão convertidas a pesos menores e a saída do algoritmo favorecerá essas regiões. Neste trabalho, o caminho mais curto é encontrado através do algoritmo de Dijkstra.

Definida a rota, a mensagem então será disseminada por meio de comunicação V2V da origem ao destino. Assim, todos os veículos pertencentes a essa rota encaminharão a mensagem. Entretanto, a fim de evitar transmissões desnecessárias e até mesmo colisões, optou-se por utilizar um mecanismo de transmissão orientado, definido por um cone (conforme Figura 3).

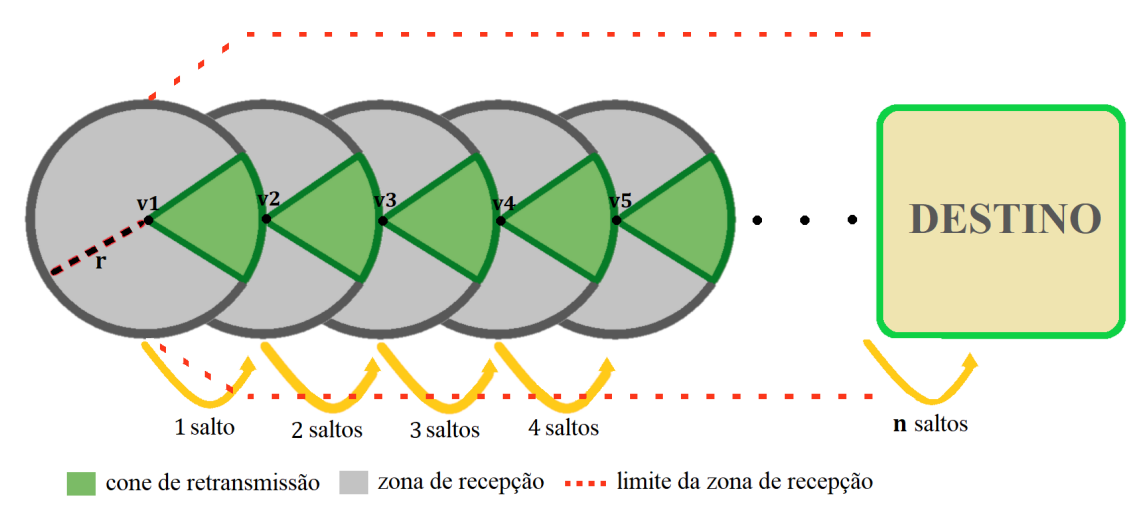

Figura 3. A retransmissão só é feita na área do cone, a cada salto até o destino da mensagem. 
Na Figura 3, vl é o veículo fonte da mensagem, Destino é a região de interesse, destino da mensagem, e $r$ é o raio de alcance do dispositivo de comunicação. Ao efetuar a transmissão da mensagem, $v 1$ alcança todos os veículos dentro do raio do dispositivo de comunicação, no entanto, define-se que apenas os veículos no interior do cone efetuarão a retransmissão das mensagens. De igual forma, os veículos que receberem a mensagem de $v 1$, conhecendo o destino da mensagem podem estimar a posição do cone e efetuarem retransmissões direcionadas. $\mathrm{O}$ ângulo de abertura do cone pode ser ajustado conforme a densidade de veículos, permitindo uma maior abrangência para densidades menores e uma menor abrangência para densidades maiores, o que contribui para mitigar o problema de broadcast storm. As retransmissões ocorrem sucessivamente, até que a mensagem alcance o destino. Conhecendo a matriz de densidades e utilizando o mecanismo de transmissão orientado ao cone de direção, o protocolo TBD define o mecanismo de retransmissão através do Algoritmo 1 .

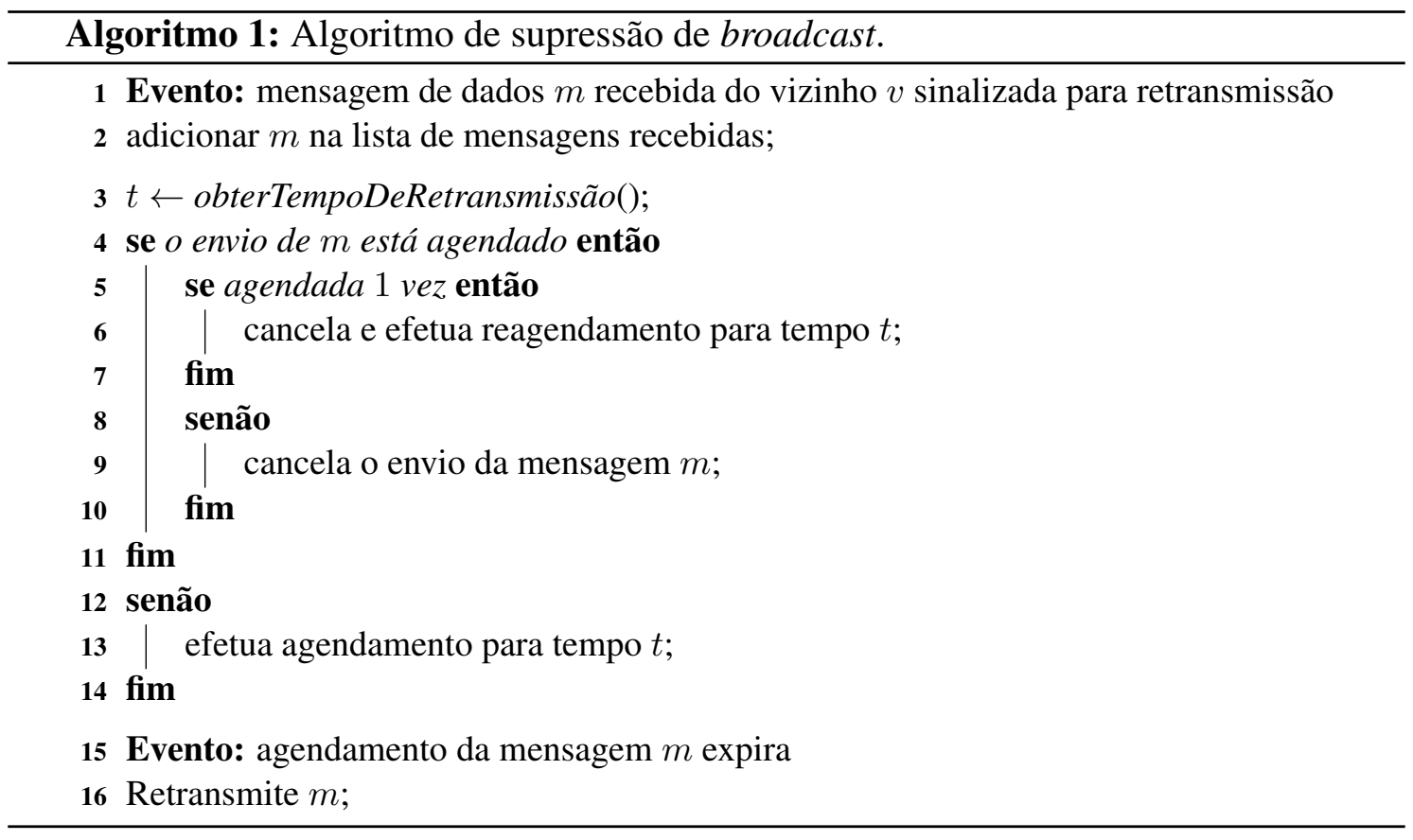

No Algoritmo 1, quando um veículo recebe uma mensagem de dados sinalizada para retransmissão (linha 1), ele deve adicionar a mensagem $m$ na lista de mensagens recebidas (linha 2). Em seguida, calcula um intervalo de tempo para retransmissão (linha 3 ) de acordo com a distância em relação ao vizinho $v$. Enquanto maior a distância, menor o tempo definido para a retransmissão. Vale destacar que se a distância for igual a $r$, a retransmissão é feita imediatamente. Com o tempo calculado, verifica-se a existência de agendamento para retransmissão futura (linha 4). Se existir e for o primeiro, cancela e efetua um reagendamento para o tempo $t$ calculado (linha 6). Caso não seja o primeiro agendamento, cancela o envio da mensagem $m$ (linha 9). Ao verificar que não existe agendamento de retransmissão da mensagem $m$, efetua o primeiro agendamento para $o$ tempo $t$ (linha 13). Assim que o tempo $t$ expira, a mensagem é então retransmitida (linhas 15 e 16). Através do mecanismo de cone de direção e deste mecanismo de supressão de broadcast, os veículos mais próximos do objetivo de levarem a mensagem ao destino na região de interesse terão prioridade na retransmissão da mensagem. 
A partir dos mecanismos apresentados, o algoritmo implementado pelo protocolo TBD toma decisão sobre a retransmissão da mensagem conforme o Algoritmo 2.

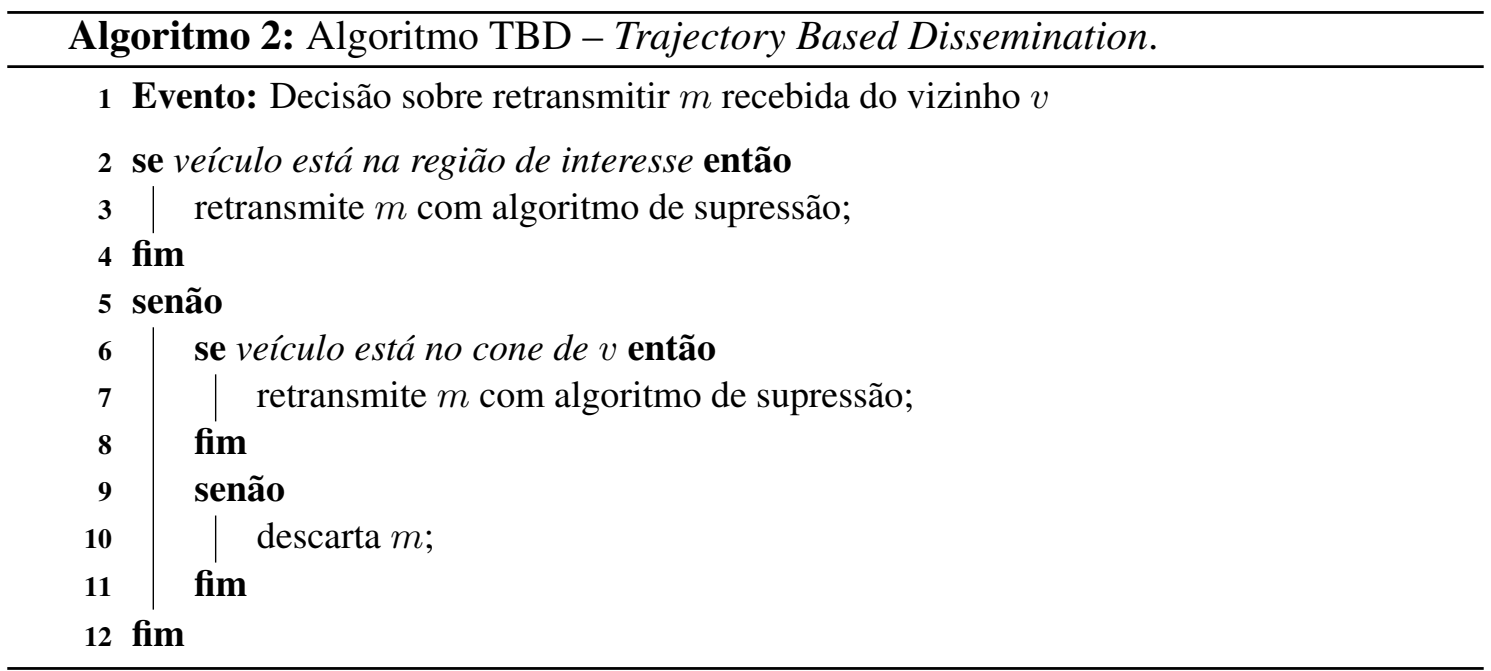

Conforme o Algoritmo 2, ao receber uma mensagem, o veículo verifica se encontrase na região de interesse (linha 2), neste caso, retransmite a mensagem por meio do Algoritmo 1 (linha 3). Caso o veículo não se encontre na região de interesse ainda, existe a necessidade de seguir uma rota para que a mensagem chegue até a região de interesse. Isso é feito verificando se o veículo está no cone de retransmissão (linha 6) do veículo que o envio. Caso esteja, o veículo efetua a retransmissão também utilizando o Algoritmo 1 (linha 7). Caso o veículo não esteja na região de interesse e também não esteja no cone, a mensagem é descartada (linha 10).

\section{Avaliação de Desempenho}

A avaliação de desempenho da solução proposta foi efetuada por meio de simulações usando como simulador de rede a ferramenta OMNeT++ 4.6 [Varga 2003] e como simulador de mobilidade a ferramenta SUMO 0.25.0 [Behrisch et al. 2011], ambos por meio do framework de redes veiculares Veins [Sommer et al. 2011]. Os resultados foram comparados com protocolos da literatura AID, CARRO e UV-CAST, apresentados na Seção 2. Estes protocolos implementam a difusão de beacons para ciência de contexto local, de maneira semelhante ao protocolo TBD que efetua submissões à rede móvel para ciência de contexto global. A seguir serão descritos os detalhes das simulações e os resultados. Na Seção 4.1 são apresentados os detalhes dos cenários de simulação bem como os parâmetros utilizados. Na Seção 4.2 são descritas as métricas usadas na avaliação, e finalmente a Seção 4.3 apresenta as discussões e os resultados de simulação.

\subsection{Cenário e Parâmetros de Simulação}

Com o objetivo de avaliar o desempenho do protocolo proposto foi implementado um cenário que favorece a ocorrência do problema de broadcast storm. Conforme a Figura 4. este é um grid de Manhattan com 20 vias duplas uniformemente espaçadas em uma área de $2000 \mathrm{~m} \times 1000 \mathrm{~m}$.

Assim, foi assumido que cada quarteirão tem $100 \times 100$ metros. Para este cenário de simulação, assumiu-se que o dispositivo de comunicação tem alcance equivalente a $250 \mathrm{~m}$, 


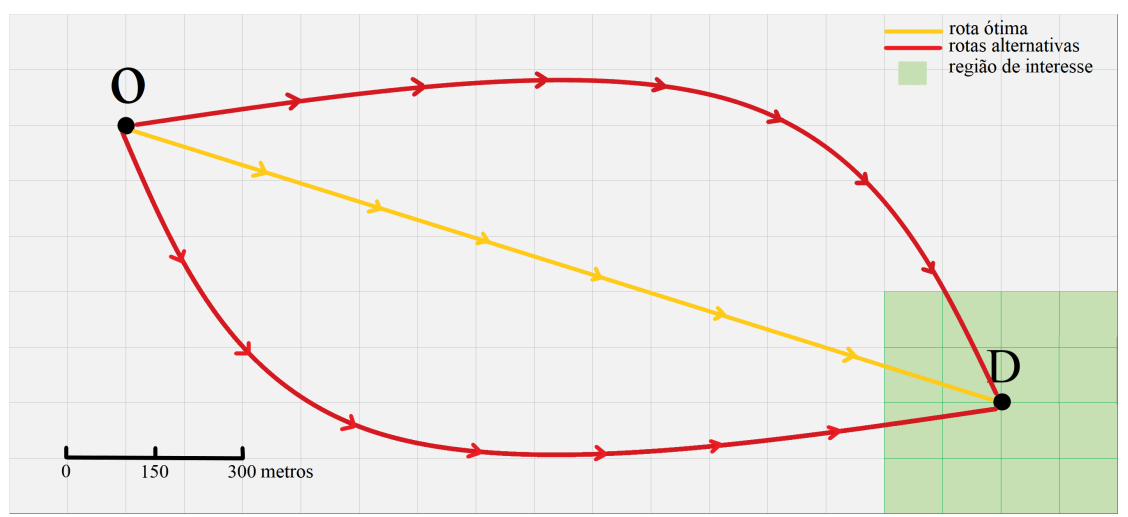

Figura 4. Cenário de Manhattan implementado, com veículo origem da mensagem numa extremidade e região de interesse na outra, o que leva aos protocolos a fazerem vários saltos até encontrarem o destino.

valor constante em $360^{\circ}$, para todos os veículos. O ângulo do cone de direção foi definido de maneira dinâmica, variando entre $110^{\circ} \mathrm{e} 130^{\circ}$, eliminando transmissões de veículos que se encontram no espaço dos $250^{\circ}$ e $230^{\circ}$ fora do cone, respectivamente. Com o objetivo de quantificar a evolução do tráfego neste cenário, a densidade de veículos foi variada de 100 veículos $/ \mathrm{km}^{2}$ a 300 veículos $/ \mathrm{km}^{2}$. O tráfego neste cenário foi gerado pelo simulador de mobilidade SUMO, e na posição $(200,200)$ do mapa foi posicionado um veículo fonte da mensagem, gerando 1 mensagem de 2048 bytes aos $15 \mathrm{~s}$ de simulação. Na outra extremidade do mapa, a região de interesse foi definida como os últimos $400 \times 400$ metros. A Tabela 2 resume os parâmetros de simulação.

\begin{tabular}{lc}
\hline \multicolumn{1}{c}{ Parâmetro } & Valor \\
\hline Tamanho do área total & $2000 \times 1000$ metros \\
Tamanho da área da ROI & $400 \times 400$ metros \\
Alcance do raio de comunicação & 250 metros \\
Ângulo do Cone dinâmico & $110^{\circ}$ até $130^{\circ}$ \\
Origem da mensagem & $(200,200)$ \\
Tamanho da mensagem & 2048 bytes \\
Freq. mensagens de localização & $1,5,9$ s. \\
Densidades avaliadas & $100-300$ veículos $/ \mathrm{km}^{2}$ \\
\hline
\end{tabular}

Tabela 2. Resumo dos parâmetros de simulação.

\subsection{Métricas}

Abaixo as métricas que foram consideradas para avaliação das soluções são apresentadas. O objetivo principal é avaliar a cobertura do protocolo, o overhead gerado pelas mensagens de dados e o atraso em diferentes condições de tráfego.

- Taxa de Entrega: O percentual de mensagens de dados gerados pelo veículo fonte e entregues a todos os veículos no cenário. O quão mais próximo de $100 \%$ for este valor, maior também será a cobertura do mapa.

- Taxa de Entrega na ROI: Representa o percentual de veículos alcançados dentro da região de interesse. O desafio nesta avaliação é garantir uma cobertura total desta região. 
- Mensagens Duplicadas: Esta métrica representa o total de mensagens repetidas recebidas pelos veículos durante o processo de disseminação.

- Percentual de Veículos Transmissores: Com esta métrica é possível avaliar o percentual de veículos envolvidos durante a disseminação. É importante destacar que uma boa solução deve garantir uma boa cobertura da ROI, evitando o overhead, i.e., mensagens transmitidas desnecessariamente. Assim, quanto menor for essa taxa, melhor será o desempenho da solução.

- Atraso médio na entrega: Esta métrica representa a média do tempo que uma mensagem de dados leva para ser transmitida da origem ao destino. Muitas aplicações em VANETs possuem restrições de atraso, por exemplo, serviços de alerta. Para estes tipos de aplicações, as mensagens devem ser disseminadas o mais rápido possível.

- Número de saltos: Esta é uma métrica que reflete o número de veículos envolvidos na entrega da mensagem, ou seja, o número de saltos entre o veículo origem e o veículo receptor da mensagem.

\subsection{Resultados}

Nesta Seção são apresentados os resultados das simulações feitas conforme parâmetros definidos na Seção 4.1. De forma a avaliar o impacto da atualização de posicionamento do protocolo TBD, os intervalos de envio de posição feitas junto à rede móvel foram definidos entre 1, 5 e 9 segundos (TBD_1, TBD_5 e TBD_9 respectivamente). Para cada uma das configurações, foram efetuados um total de 50 replicações. Os resultados apresentados a seguir representam a média dos valores obtidos com essas replicações.

Os gráficos da Figura 5 apresentam os resultados de simulações do ponto de vista do número de mensagens disseminadas. No gráfico da Figura 5 -(a), temos a taxa de entrega das disseminações efetuadas, incluindo os veículos que não estão no interior da região de interesse. Pode-se observar que a porcentagem total de veículos alcançados pelo TBD é inferior aos demais protocolos avaliados. Isso se deve ao fato de a mensagem percorrer um caminho no mapa, levando a mensagem até o destino, transmitindo o mínimo aos veículos que não são parte do caminho e que não estão na região de interesse. Observa-se que os protocolos CARRO, AID e UV-CAST apresentam uma taxa de entrega bem maior.

Ao variar a densidade da rede, observa-se que os demais protocolos mantêm a proporção de veículos alcançados. No entanto, o TBD mantém a taxa de recepção da mensagem apenas no caminho da disseminação e na região de interesse, ou seja, o número de mensagens recebidas total para o protocolo TBD será sempre menor e mais perceptível enquanto maior for o cenário. Nota-se também que as três versões apresentam o comportamento semelhantes, com pouca variação quando a densidade de veículos é aumentada.

O gráfico da Figura 5-(b) apresenta a taxa de entrega no interior da região de interesse. Importante ressaltar que nos cenários avaliados, o tamanho da região de interesse representa aproximadamente $10 \%$ de toda a rede. Pode-se observar que os protocolos UVCAST, AID e TBD com atualizações de densidade a cada $1 \mathrm{~s}$. apresentam comportamento semelhante, entregando a mensagem em toda a região de interesse. Em razão do maior intervalo entre atualizações de densidade, o protocolo TBD com atualizações a cada 5 e a cada 9 segundos sofrem um pouco para entregarem na região interesse. Isso ocorre devido 
a falta de precisão nas informações de localização, o que pode gerar atrasos e gargalos durante o encaminhamento da mensagem até a região.

O gráfico da Figura 5-(c) apresenta o número de mensagens duplicadas por veículos em todo o mapa. É possível observar que o protocolo TBD apresenta os melhores resultados, limitando o número de mensagens transmitidas a poucos veículos. De acordo com o seu funcionamento que define um cone que direciona a retransmissão das mensagens, a quantidade de veículos retransmissores é reduzida. Mesmo com informações menos precisas, o protocolo TBD com atualizações a cada 5 e 9 segundos reduzem o número de mensagens duplicadas a metade, comparando com os demais protocolos. Nota-se também que os demais protocolos, por não implementarem nenhum mecanismo de contenção na disseminação, apresentaram valores bem superiores, destacando CARRO e AID com as maiores taxas.

Ao observar a taxa de veículos transmissores no gráfico da Figura 5-(d), pode-se observar conformidade com os resultados já apresentados. A quantidade de veículos envolvidos na disseminação pelo protocolo TBD é inferior em todas as densidades, e reduz conforme a densidade de veículos aumenta. Ao comparar com as demais soluções avaliadas, pode-se verificar que cerca de $25 \%$ dos veículos estão envolvidos na disseminação dos dados, o que explica os resultados obtidos com as mensagens duplicadas.

Os gráficos da Figura 6 apresentam resultados das simulações do ponto de vista da eficiência na entrega e do número de saltos. Para todos os protocolos foi definido que o maior tempo de espera para uma retransmissão é 500ms. Esta escolha acompanha o trabalho de [Viriyasitavat et al. 2010], em que o autor mostra que o atraso deve ser cuidadosamente escolhido, e o valor $500 \mathrm{~ms}$ pode ser definido como limite superior. Para o autor, o assunto deve ser mais estudado em trabalhos futuros.

Pode-se observar os resultados para o atraso médio na Figura 6-(a). Nota-se que o protocolo TBD apresenta resultados bastante satisfatórios comparado aos demais da literatura, igualando ao protocolo AID que também apresenta um atraso baixo. Tanto o protocolo CARRO e UV-CAST adicionam atraso nas retransmissões como mecanismo de contenção para o problema de broadcast storm. Por esse motivo esses protocolos apresentam valores superiores. Ao analisar o comportamento do TBD, pode-se notar um bom desempenho do protocolo em todas as suas três versões, independente da densidade de veículos avaliada.

Ao analisar o número médio de saltos efetuados na disseminação (Figura6-(b)), pode-se notar que em média o TBD apresenta 2 saltos a menos que os demais. $\mathrm{O}$ atraso ser inferior está diretamente ligado à quantidade de saltos da origem ao destino da mensagem, assim, por apresentar um número de saltos inferior, o protocolo TBD apresenta melhores resultados em termos de atraso.

Além dos resultados apresentados sob as métricas definidas na Seção 4.2, percebeuse que o intervalo de tempo entre os acessos à rede celular para submissão das novas posições pouco influenciou o desempenho do protocolo. Esta informação sugere que conforme o tempo passa, as densidades de veículos no mapa não sofrem mudanças significativas em relação aos segundos anteriores devido aos veículos estarem limitados pelas restrições espaciais. Essa informação contribui com a avaliação de custo financeiro dos acessos à rede móvel, sugerindo que são desnecessários acessos muito frequentes devido a 


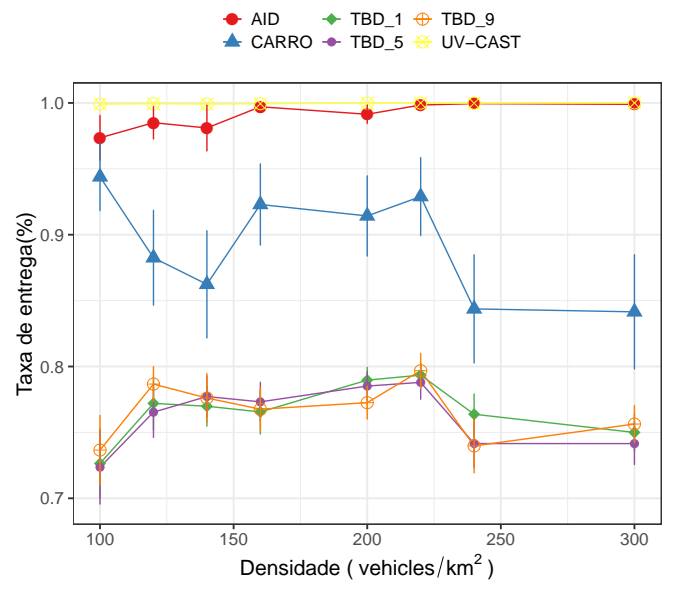

(a) Taxa de Entrega.

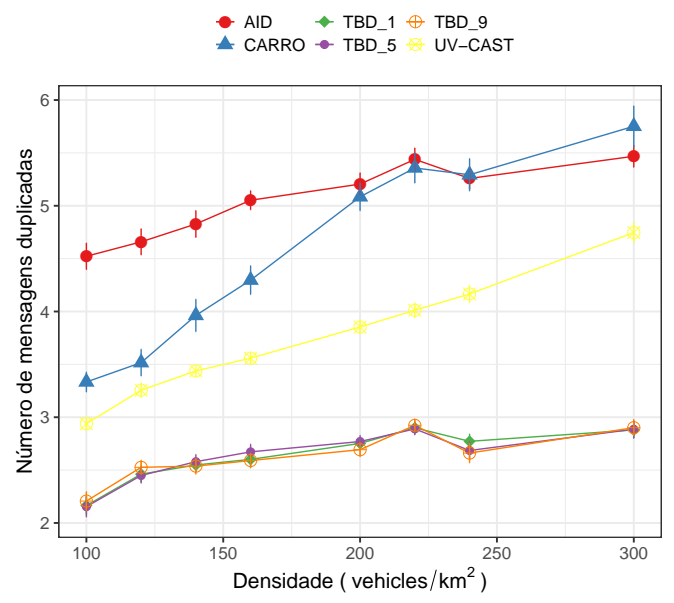

(c) Mensagens Duplicadas.

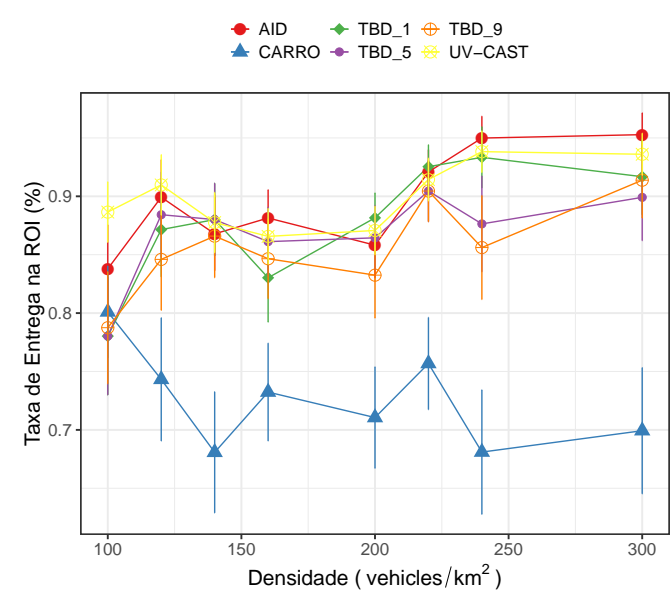

(b) Taxa de Entrega na ROI.

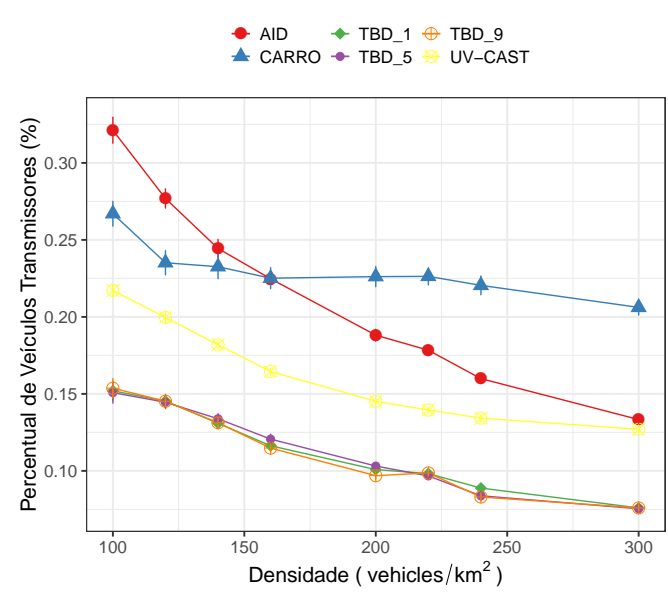

(d) Veículos Transmissores.

Figura 5. Resultados de simulação considerando o comportamento das mensagens enviadas e recebidas.

não agregarem informações capazes de refletir em desempenho.

\section{Conclusão e Trabalhos Futuros}

Neste trabalho foi proposto um protocolo de disseminação de dados que levasse a mensagem da origem até a região de interesse e na região de interesse entregasse a todos os veículos. Para este fim, o protocolo proposto utilizou a infraestrutura existente nas cidades, a rede celular, como forma de comunicação de controle. Cada veículo atualiza sua posição via rede celular periodicamente, permitindo que os demais veículos da cidade conheçam as densidades de veículos de cada região. Através desse conhecimento, o protocolo TBD faz com que a mensagem seja transmitida por um caminho de densidades, de forma que a mensagem a ser disseminada na região de interesse não seja entregue e retransmitida por veículos fora da região de interesse ou fora do caminho da disseminação. Os resultados 


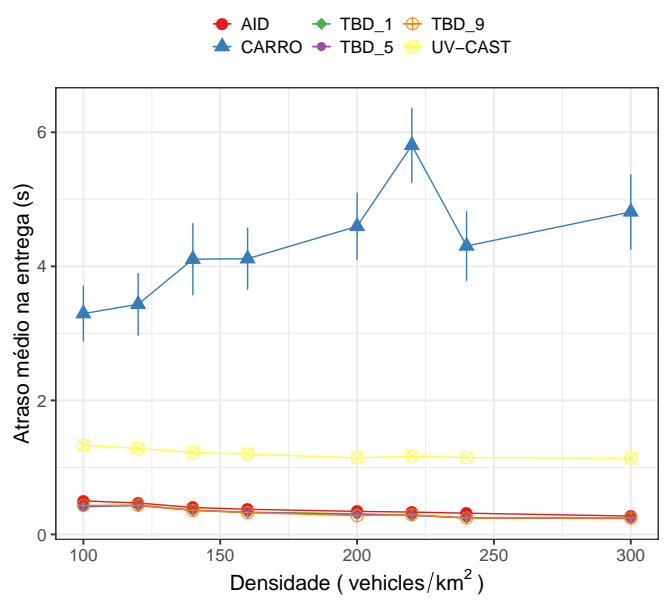

(a) Atraso Médio.

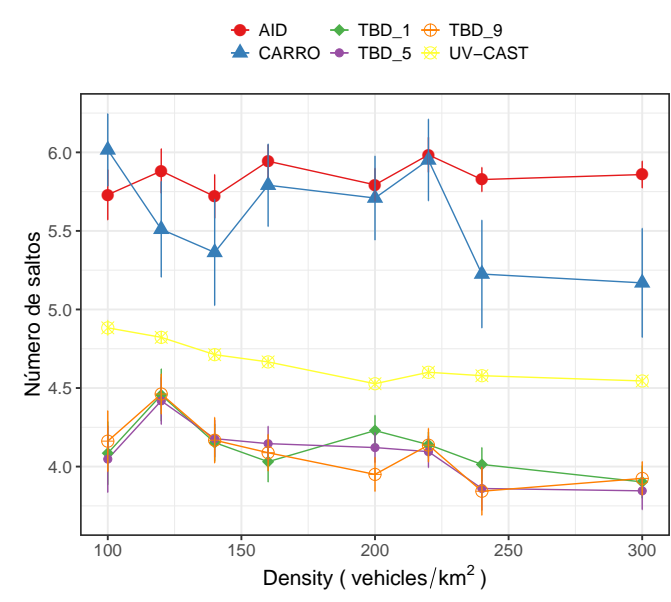

(b) Saltos

Figura 6. Comparação do número de saltos e atraso no recebimento das mensagens.

de simulações mostraram que o protocolo desempenha bem para o problema proposto, reduzindo de maneira expressiva o número de mensagens duplicadas, bem como o número de veículos não interessados na mensagem que a recebem. Foi verificado também que o percentual de veículos transmissores diminuiu, o que explica a redução no número de mensagens duplicadas, sem mudança significativa na entrega aos veículos no interior da região de interesse. Além disso, foi observado que o intervalo entre atualizações pouco interfere nos resultados de entrega e custo de transmissões.

Para trabalhos futuros, pretende-se utilizar do conhecimento dos veículos sobre seus destinos e velocidades para tornar as atualizações à rede celular dinâmicas, sensíveis às informações locais de cada veículo na rede. Essas informações permitirão a tomada de decisão mais inteligente acerca do intervalo de tempo entre as atualizações de posição, o que pode reduzir o número de acessos à rede. Além disso, pretende-se avaliar novas formas para definição da trajetória, reduzindo a quantidade de veículos transmissores sem comprometer a cobertura da disseminação na região de interesse.

\section{Referências}

[Akabane et al. 2016] Akabane, A. T., Pazzi, R. W., Madeira, E. R., and Villas, L. A. (2016). Carro: A context-awareness protocol for data dissemination in urban and highway scenarios. In Communications (LATINCOM), 2016 8th IEEE Latin-American Conference on, pages 1-6. IEEE.

[Bakhouya et al. 2011] Bakhouya, M., Gaber, J., and Lorenz, P. (2011). An adaptive approach for information dissemination in vehicular ad hoc networks. Journal of Network and Computer Applications, 34(6):1971-1978.

[Behrisch et al. 2011] Behrisch, M., Bieker, L., Erdmann, J., and Krajzewicz, D. (2011). Sumo-simulation of urban mobility. In The Third International Conference on Advances in System Simulation (SIMUL 2011), Barcelona, Spain. 
[Costa et al. 2017] Costa, J., Lobato, W., de Souza, A. M., Rosário, D., Villas, L. A., and Cerqueira, E. (2017). Centrality-based data dissemination protocol for vehicular ad hoc networks. In 2017 IEEE 16th International Symposium on Network Computing and Applications (NCA), pages 1-4.

[Cunha et al. 2014] Cunha, F. D., Maia, G. G., Viana, A. C., Mini, R. A., Villas, L. A., and Loureiro, A. A. (2014). Socially inspired data dissemination for vehicular ad hoc networks. In Proceedings of the 17th ACM international conference on Modeling, analysis and simulation of wireless and mobile systems, pages 81-85. ACM.

[Maia et al. 2013] Maia, G., Villas, L. A., Boukerche, A., Viana, A. C., Aquino, A. L. L., and Loureiro, A. A. F. (2013). Data dissemination in urban vehicular ad hoc networks with diverse traffic conditions. In 2013 IEEE Symposium on Computers and Communications (ISCC), pages 000459-000464.

[Maia et al. 2015] Maia, G., Villas, L. A., Viana, A. C., Aquino, A. L., Boukerche, A., and Loureiro, A. A. (2015). A rate control video dissemination solution for extremely dynamic vehicular ad hoc networks. Performance Evaluation, 87:3-18.

[Peng et al. 2018] Peng, H., Liang, L., Shen, X., and Li, G. Y. (2018). Vehicular communications: A network layer perspective. IEEE Transactions on Vehicular Technology, pages $1-1$.

[Sommer et al. 2011] Sommer, C., German, R., and Dressler, F. (2011). Bidirectionally Coupled Network and Road Traffic Simulation for Improved IVC Analysis. IEEE Transactions on Mobile Computing, 10(1):3-15.

[Tonguz et al. 2010] Tonguz, O. K., Wisitpongphan, N., and Bai, F. (2010). Dv-cast: A distributed vehicular broadcast protocol for vehicular ad hoc networks. IEEE Wireless Communications, 17(2):47-57.

[Toor et al. 2008] Toor, Y., Muhlethaler, P., Laouiti, A., and Fortelle, A. D. L. (2008). Vehicle ad hoc networks: applications and related technical issues. IEEE Communications Surveys Tutorials, 10(3):74-88.

[Tseng et al. 2002] Tseng, Y.-C., Ni, S.-Y., Chen, Y.-S., and Sheu, J.-P. (2002). The broadcast storm problem in a mobile ad hoc network. Wireless networks, 8(2-3):153-167.

[Varga 2003] Varga, A. (2003). Omnet++ simulator. Department of Telecommunications, Technical University of Budapest, Budapest, Hungary.

[Villas et al. 2012] Villas, L. A., Ramos, H. S., Boukerche, A., Guidoni, D. L., Araujo, R. B., and Loureiro, A. A. (2012). An efficient and robust data dissemination protocol for vehicular ad hoc networks. In Proceedings of the 9th ACM symposium on Performance evaluation of wireless ad hoc, sensor, and ubiquitous networks, pages 39-46. ACM.

[Viriyasitavat et al. 2010] Viriyasitavat, W., Bai, F., and Tonguz, O. K. (2010). Uv-cast: an urban vehicular broadcast protocol. In Vehicular Networking Conference (VNC), 2010 IEEE, pages 25-32. IEEE.

[W.H.O. 2013] W.H.O. (2013). Global status report on road safety 2013: supporting a decade of action: summary. World Health Organization. 